\title{
THCCOOH concentrations in whole blood: Are they useful in discriminating occasional from heavy smokers?
}

\author{
Marie Fabritius, ${ }^{a}$ Bernard Favrat, ${ }^{b}$ Haithem Chtioui, ${ }^{c}$ Giovanni Battistella, ${ }^{d}$ \\ Jean-Marie Annoni, ${ }^{\mathrm{e}}$ Monique Appenzeller, ${ }^{\mathrm{C}} \mathrm{Kim}^{\mathrm{D}}$ Dao, ${ }^{\mathrm{c}}$ Eleonora Fornari, ${ }^{\mathrm{d}, \mathrm{f}}$ \\ Estelle Lauer, ${ }^{a}$ Jean-Frédéric Mall, ${ }^{9}$ Philippe Maeder, ${ }^{d}$ Patrice Mangin, ${ }^{a}$ \\ Christian Staub ${ }^{a}$ and Christian Giroud ${ }^{a_{*}}$
}

\begin{abstract}
Some forensic and clinical circumstances require knowledge of the frequency of drug use. Care of the patient, administrative, and legal consequences will be different if the subject is a regular or an occasional cannabis smoker. To this end, 11-nor-9-carboxy- $\Delta$ 9-tetrahydrocannabinol (THCCOOH) has been proposed as a criterion to help to distinguish between these two groups of users. However, to date this indicator has not been adequately assessed under experimental conditions. We carried out a controlled administration study of smoked cannabis with a placebo. Cannabinoid levels were determined in whole blood using tandem mass spectrometry. Significantly high differences in $\mathrm{THCCOOH}$ concentrations were found between the two groups when measured during the screening visit, prior to the smoking session, and throughout the day of the experiment. Receiver operating characteristic (ROC) curves were determined and two threshold criteria were proposed in order to distinguish between these groups: a free THCCOOH concentration below $3 \mu \mathrm{g} / \mathrm{L}$ suggested an occasional consumption $(\leq 1$ joint/week) while a concentration higher than $40 \mu \mathrm{g} / \mathrm{L}$ corresponded to a heavy use ( $\geq 10$ joints/month). These thresholds were tested and found to be consistent with previously published experimental data. The decision threshold of $40 \mu \mathrm{g} / \mathrm{L}$ could be a cut-off for possible disqualification for driving while under the influence of cannabis. A further medical assessment and follow-up would be necessary for the reissuing of a driving license once abstinence from cannabis has been demonstrated. A THCCOOH level below $3 \mu \mathrm{g} / \mathrm{L}$ would indicate that no medical assessment is required.
\end{abstract}

Keywords: $\mathrm{THCCOOH}$; cannabis; whole blood; heavy use; occasional use

\section{Introduction}

Cannabis is the most widely used illegal drug in Switzerland and in the western world. According to Addiction Monitoring Switzerland $2012^{[1]} 5.1 \%$ of the Swiss population aged 15 or older has used cannabis at least once during the last 12 months $(7.5 \%$ of men, $2.8 \%$ of women). The first nationwide study on driving while under the influence of drugs in Switzerland confirmed that the most frequently encountered drugs in whole blood (WB) were cannabinoids (48\% of total number of cases). ${ }^{[2]} \Delta 9$ tetrahydrocannabinol (THC), together with its inactive metabolite 11-nor-9-carboxy-THC (THCCOOH), was frequently detected in the blood of impaired drivers arrested for careless driving or involved in road accidents. ${ }^{[3,4]}$ Accurate measurement and interpretation of cannabinoid concentrations can contribute to the reconstruction of the accident scene, determining the time lapse between the last cannabis use and the accident and the assessment of driving impairment level. In this regard, the ability to drive can be affected in the short term, long term, or permanently. The fitness to drive (long term) can be influenced by many parameters. One of them is frequency of cannabis use. Several recent studies suggest that, compared to occasional users, regular smokers experience the typical desired and adverse effects of cannabis less intensely. In the few controlled administration studies carried out so far with heavy cannabis smokers, ${ }^{[5,6]}$ blunted acute psychological and psychomotor disturbances have been reported. Therefore, the development of a specific test and criterion to distinguish regular cannabis use $(\geq 10$ joints per

* Correspondence to: Christian Giroud, CURML (University Center of Legal Medicine), UTCF (Forensic Toxicology and Chemistry Unit), Lausanne and Geneva, Switzerland.E-mail: christian.giroud@chuv.ch

a CURML (University Center of Legal Medicine), UTCF (Forensic Toxicology and Chemistry Unit), Lausanne and Geneva, Switzerland

b CURML (University Center of Legal Medicine), UMPT (Unit of Psychology and Traffic Medicine), Lausanne and Geneva, Switzerland

c Division of Clinical Pharmacology and Toxicology, Centre Hospitalier Universitaire Vaudois (CHUV), Lausanne, Switzerland

d Department of Radiology, Centre Hospitalier Universitaire Vaudois (CHUV), Lausanne, Switzerland

e Neurology Unit, Department of Medicine, University of Fribourg, Fribourg, Switzerland

f CIBM (Centre d'imagerie BioMédicale), Centre Hospitalier Universitaire Vaudois (CHUV) unit, Lausanne, Switzerland

g Department of Psychiatry, SUPAA (Service Universitaire de Psychiatrie de l'Age Avancé), Centre Hospitalier Universitaire Vaudois (CHUV), Lausanne, Switzerland 
month) from occasional consumption ( $\leq 1$ joint per week) is required to perform relevant driving ability evaluations. The suspicion of chronic consumption triggers a cascade of heavy and expensive administrative measures, but also of toxicological investigations to confirm a long-term alteration of the fitness to drive. If the assumption of regular cannabis use is refuted, the driver could reclaim his confiscated driving licence. The subject's medical history combined with a medical examination is only of limited use in detecting chronic cannabis use. In general, it is preferable to rely on objective data. Regular consumption can be assessed by measuring drug concentrations in hair. However, this matrix is not always available, or can be contaminated by cannabis smoke, ${ }^{[7]}$ or by contact with contaminated body parts (e.g. urine contaminated dirty hands) and materials, which makes the interpretation of analytical results difficult. Furthermore, in comparative studies, discrepancies have been found between 'positive' urine specimens and hair tested as 'negative ${ }^{[8,9]}$ More recently, Han et al. failed to detect a significant correlation between self-reported doses and frequency of use and low, medium, and high hair $\mathrm{THCCOOH}$ concentration range groups. ${ }^{[10,11]}$ If hair analysis is of limited value in estimating the frequency of cannabis consumption, it is, however, widely used in the context of abstinence control. Thus, the measurement of cannabinoids in blood offers another strategy to assess the frequency of cannabis use. In this context, Daldrup et al. ${ }^{[12]}$ have suggested that if the serum concentration of $\mathrm{THCCOOH}$ is lower than $5 \mu \mathrm{g} / \mathrm{L}$ the consumption is assumed to be occasional, while levels above $75 \mu \mathrm{g} / \mathrm{L}$ are associated with regular use. This criterion could be applied in routine forensic examinations on the condition that serum samples are taken within an eight-day period following the last cannabis use. ${ }^{[9]}$ Assuming that the smoker would have stopped smoking during the eight days prior to blood sampling, the concentration of $\mathrm{THCCOOH}$ at the relevant time should be doubled to $150 \mu \mathrm{g} / \mathrm{L}$. In 2008, Toennes et al. ${ }^{[13]}$ compared the pharmacokinetic properties of the three main cannabinoids in occasional and heavy cannabis users. They reported much higher concentrations in the serum collected from heavy users than from occasional smokers. These authors also indicated that the concentration ranges for these two consumer groups greatly overlapped. In 2013, the French Society of Analytical Toxicology (SFTA) advocated the use of hair analysis to discriminate between occasional and regular cannabis use. ${ }^{[14]}$ On the other hand, the SFTA recommended a cut-off of $50 \mathrm{ng} / \mathrm{mL}$ of $\mathrm{THCCOOH}$ WB for the inferior limit, over which a regular consumption of cannabis (1 joint/day, each day, or more) could be strongly suspected. In this study, we report on $\mathrm{THCCOOH}$ concentrations measured in 797 WB specimens collected during a placebo-controlled smoking administration study of cannabis in which heavy and occasional subjects were enrolled. WB samples were collected during the screening visit, before and after smoking a joint or a placebo, and up to $3.5 \mathrm{~h}$ after the first puff. THCCOOH levels in WB were determined by gas chromatography (GC) or liquid chromatography (LC) combined with tandem mass spectrometry (MS/MS).

The purpose of this study is to show that significant differences between these two populations of smokers exist. The second goal is to propose two $\mathrm{THCCOOH}$ threshold concentrations to help determine whether they are occasional or regular cannabis consumers.

\section{Materials and methods}

\section{Study design}

The overall design of the study that included various clinical, neuroimaging, and psychomotor investigations has been described in a previous publication and its supporting information. ${ }^{[15]}$ The imaging sessions determined the timeframe of the one-day experiment. The study was extended to include a similar-sized group of young male heavy cannabis smokers. Twenty-three heavy smokers and 25 occasional smokers, between 18 and 30 years of age, participated in this study. For the occasional smokers, the mean consumption of cannabis during the three months preceding inclusion in the study was set at a minimum of one joint per month and a maximum of one joint per week. It was set at a minimum of ten joints per month and less than three joints per day for the heavy users. Heavy smokers who were multi-daily users ( $>3$ joints/day) were excluded from the study because they were unlikely to remain drug-free during the experimental day, mostly after placebo smoking. Main sociodemographic characteristics and habits of cannabis consumption reported by the volunteers are presented in Table 1. Consumption of any illegal drug other than cannabis was an exclusion criterion from the study. Subjects recruited for the study participated in two independent cross-over experimental sessions in which they smoked either a joint of pure cannabis $(0.7 \mathrm{~g}$ Bedrobinol, $11 \%$ THC, < 1\% CBD, purchased from Bedrocan, Veendam, the Netherlands) or a placebo $(0.8 \mathrm{~g}$ Santhica variety, no THC, $<0.1 \%$ $\mathrm{CBD}$, provided by the French National Federation of Hemp Growers, FNPC, Le Mans, France). The fixed-paced smoking procedure consisted of $3 \mathrm{~s}$ of preparation, $2 \mathrm{~s}$ of inhalation, $5 \mathrm{~s}$ of breath holding and $50 \mathrm{~s}$ of exhalation and rest. This sequence was repeated until two-thirds of the joint was consumed, up to a line drawn $3 \mathrm{~cm}$ above the cardboard filter. The residue was weighed to estimate the smoked dose. ${ }^{[15]}$ The investigation period began

\begin{tabular}{|c|c|c|c|c|c|c|}
\hline & Number & Mean & SD & Median & Maximum & Minimum \\
\hline \multicolumn{7}{|l|}{ Occasional smokers } \\
\hline Age & & 23.9 & 3.0 & 24 & 29 & 19 \\
\hline Age at first cannabis use & & 16.3 & 2.9 & 16 & 23 & 9 \\
\hline Frequency of use (occasions/month, last three months) & & 3.5 & 2.3 & 3.5 & 10 & 1 \\
\hline Driving license & \multicolumn{2}{|c|}{$22 / 25(88 \%)$} & & & & \\
\hline \multicolumn{7}{|l|}{ Heavy smokers } \\
\hline Age & & 22.7 & 2.4 & 22 & 28 & 19 \\
\hline Age at first cannabis use & & 16.3 & 2.3 & 16 & 20 & 12 \\
\hline Frequency of use (occasions/month, last three months) & & 77.9 & 51.7 & 62.5 & 250 & 20 \\
\hline Driving license & \multicolumn{2}{|c|}{ 14/23 (61\%) } & & & & \\
\hline
\end{tabular}


in the late morning (11 a.m.) and ended in the evening when the symptoms of intoxication were almost imperceptible (up to 9 p.m.). The volunteers were kept under close watch throughout the day of the experiment. Blood samples were collected several hours $(5 \mathrm{~h})$ and a few minutes before smoking the joint $(\mathrm{t}=0 \mathrm{~h})\left(\mathrm{C}_{0}\right)$ and at $0.2,0.3,0.4,0.65,1.9,2.5$, and $3.5 \mathrm{~h}$ after inhalation. Samples were immediately frozen and kept at $-80^{\circ} \mathrm{C}$ before analysis. For time schedule reasons, the last blood sample was omitted for the occasional smokers.

This study was conducted in accordance with good clinical practices and was approved by the Cantonal Research Ethics Committee (Vaud). The subjects gave written informed consent and received financial compensation for their participation.

\section{Materials}

THC, THCCOOH, and corresponding internal standards (THC- $\mathrm{d}_{3}$ and $\mathrm{THCCOOH}-\mathrm{d}_{9}$ ) were obtained from Cerilliant Corporation (Round Rock, TX, USA). Trifluoroacetic anhydride (TFAA) and hexafluoro-2-propanol (HFIP) were purchased from Supelco (Bellefonte, PA, USA) and Fluka (Buchs, Switzerland), respectively. Hexane, acetonitrile and ethyl acetate were of high-performance chromatographic grade from Merck (Darmstadt, Germany).

\section{Methods}

Analyses of the occasional smokers' blood specimens were carried out according to the procedure described by Thomas et $a{ }^{[16]}$ Five hundred $\mu \mathrm{L}$ of WB were extracted using a liquid-liquid extraction (LLE) with hexane: ethyl acetate (9:1, $v / v)$ and then derivatized with a mixture of TFAA and HFIP as fluorinated agents. The analysis by MS was performed in the selected reaction-monitoring mode with a Varian $1200 \mathrm{~L}$ MS/MS triple quadrupole instrument after negative-ion chemical ionization. The limits of quantification (LOQ) of the method were $0.5 \mu \mathrm{g} / \mathrm{L}$ and $2.5 \mu \mathrm{g} / \mathrm{L}$ for THC and $\mathrm{THCCOOH}$, respectively.

The same extraction procedure was used for the blood samples of the heavy smokers, but the analysis was carried out by LC-MS/ MS without a derivatization step. The ion transitions, MS parameters and LC-MS/MS equipment were the same as those described in a previously published procedure for oral fluid. ${ }^{[17]}$ The recommendations of the French Society of Pharmaceuticals Sciences and Techniques (SFSTP) were followed to validate this LC-MS/MS method. Linearity of this method was from 0.5 to $20 \mu \mathrm{g} / \mathrm{L}$ and from 2.5 to $100 \mu \mathrm{g} / \mathrm{L}$ for THC and THCCOOH, respectively. Four quality control specimens (QC) $(0.5,1,10,20 \mu \mathrm{g} / \mathrm{L}$ for $\mathrm{THC}$ and 2.5, 10, 50, $100 \mu \mathrm{g} / \mathrm{L}$ for $\mathrm{THCCOOH}$ ) were analyzed four times on the same day and on three non-consecutive days to determine the trueness and precision parameters. ${ }^{[16]}$ The trueness results ranged between $86 \%$ and $113 \%$. The coefficients of variation (CV) of intra-day and intermediate precisions were lower than $15 \%$ in all cases. Intraday precision results ranged between 3.5 and $11 \%$ for THC and between 5.8 and $9.8 \%$ for $\mathrm{THCCOOH}$. Intermediate precision values ranged between 3.8 and $9.8 \%$ and 3.9 and $13.8 \%$ for $\mathrm{THC}$ and $\mathrm{THCCOOH}$, respectively. $\mathrm{LOQ}$ values were determined at the lower QC with a trueness higher than $85 \%$ and CVs lower than 20\%. LOQs were 0.5 and $2.5 \mu \mathrm{g} / \mathrm{L}$ for $\mathrm{THC}$ and $\mathrm{THCCOOH}$, respectively. To facilitate the reading of the results, concentrations lower than the LOQ were reported as zero. To cross-validate GC-MS/MS and LC-MS/MS methods, the same QC were analyzed. No significant difference was observed between these methods. Furthermore, ten authentic blood specimens were analyzed with both methods yielding almost identical results.

\section{Statistical analysis}

Data, receiver operating characteristics (ROC) curves, box and whisker graphs were processed and plotted using Excel 2007 and GraphPad Prism 6.02 softwares. True positives (TP) were correctly identified smokers, i.e. heavy smokers with $\mathrm{THCCOOH}$ concentrations higher than the threshold. False positives (FP) were incorrectly identified heavy smokers, i.e. occasional smokers with $\mathrm{THCCOOH}$ concentrations higher than the threshold. True negatives (TN) were correctly rejected consumers, i.e. occasional smokers with $\mathrm{THCCOOH}$ concentrations lower than the threshold. False negatives (FN) were incorrectly rejected users, i.e. heavy smokers with $\mathrm{THCCOOH}$ levels lower than the threshold. Sensitivity was the proportion of true positives in heavy smokers who presented sample values exceeding the threshold and was calculated as TP / TP + FN. Specificity was estimated by the ratio: TN / TN + FP. The positive likelihood ratio $(\mathrm{LR}+)$ was calculated as follows: sensitivity divided by 1 - specificity. ${ }^{[18,19]}$ The ROC curve is a graphical plot of true positive rate (sensitivity) versus false positive rate (1-specificity) for each $\mathrm{THCCOOH}$ threshold. The maximum specificity cut-off to rule in the heavy users was set close to the lower left corner of the ROC curve while the maximum sensitivity cut-off to rule out the heavy smokers was set close to the upper right corner.

\section{Results}

\section{Sociodemographic characteristics of the volunteers}

The two groups of young men were very similar: 25 were occasional smokers and 23 were heavy cannabis users. Their mean age were almost the same (23.9 and 22.7, respectively). Surprisingly, they had the same age of first cannabis use (16.3), but they differed in their frequency of consumption (3.5 and 77.9 occasions per month for occasional and heavy smokers, respectively). A higher proportion of occasional consumers had a valid driving license (88 versus $61 \%$ ).

\section{Kinetic profiles of THC and THCCOOH}

Figure 1 shows the median time profiles of $\mathrm{THC}$ and $\mathrm{THCCOOH}$ for the heavy and occasional smokers, before and after smoking a cannabis joint. These consumers presented similar profiles with parallel kinetic curves. When considering each curve separately, the inter-quartile range indicates a broad but relatively constant dispersion of $\mathrm{THCCOOH}$ values throughout the experiment timeframe. Median $C_{0}$ were 2.1 (range 0.8 to 7.1 ) and $0(0-1.4)$ $\mu \mathrm{g} / \mathrm{L}$ for THC and $20(2.5-51)$ and $0(0-17) \mu \mathrm{g} / \mathrm{L}$ for $\mathrm{THCCOOH}$ for the heavy and occasional cannabis users, respectively. This latter difference of about $20 \mu \mathrm{g} / \mathrm{L}$ between the two $\mathrm{THCCOOH}$ $C_{0}$ levels remained more or less constant throughout the experience: for example, median $\mathrm{THCCOOH}$ concentrations measured $2.5 \mathrm{~h}$ after smoking the joint were 26 (9.4-78) and 7.1 $(0-29) \mu \mathrm{g} / \mathrm{L}$ for the heavy and occasional smokers respectively. Cannabinoid levels were also determined before and after smoking the placebo joint (results not shown). The median THC levels remained lower than $2 \mu \mathrm{g} / \mathrm{L}$ throughout the experiment, while $\mathrm{THCCOOH}$ concentrations in the heavy smokers were 


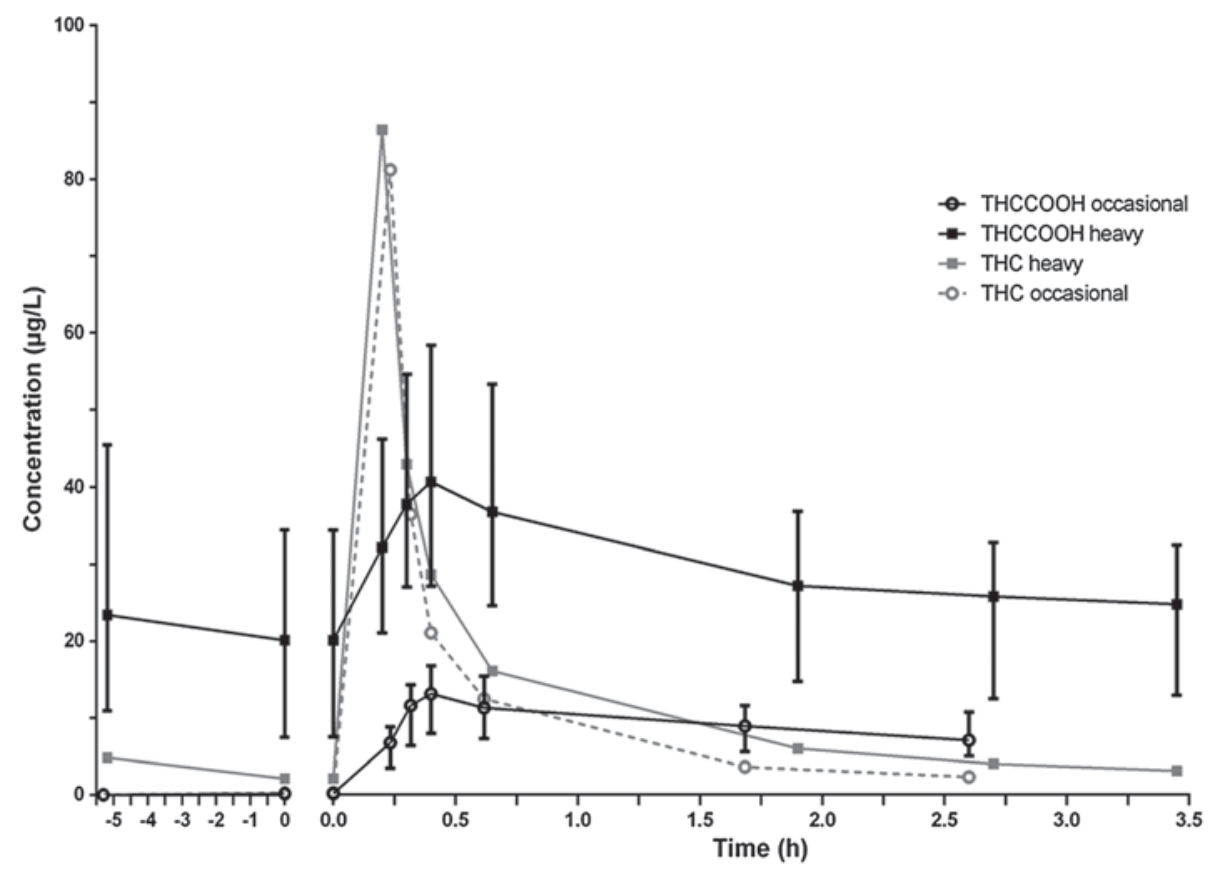

Figure 1. Median THC and median (interquartile) THCCOOH time profiles in whole blood of occasional and heavy users before and after smoking a cannabis joint.

almost all significantly higher than the LOQ value. The median $\mathrm{THCCOOH}$ concentrations in the heavy smokers decreased slowly from $17(4.3-48)$ to $10(0-32) \mu \mathrm{g} / \mathrm{L}$ between $5 \mathrm{~h}$ before and $3 \mathrm{~h}$ after smoking the placebo joint. The corresponding $C_{0}$ median level was $12(2.9-38) \mu \mathrm{g} / \mathrm{L}$. THC could not be quantified in any sample of occasional users after smoking the placebo. $\mathrm{THCCOOH}$ was detected in only 14 samples out of 393, i.e. the median value at each time point remains 0 during the placebo experimental session.

\section{Concentration differences for $\mathrm{THCCOOH}$ between occasional and regular cannabis smokers}

The results for $\mathrm{THCCOOH}$ levels are presented as box and whisker plots in Figure 2. Statistical comparisons (Mann-Whitney test) were made between the occasional and heavy smokers at each time point. The heavy smokers' $\mathrm{THCCOOH}$ concentrations were also compared to the Cmax values of the occasional smokers. Median (range) $\mathrm{THCCOOH}$ concentrations determined at inclusion were $24(4.6-70)$ and $0(0-<2.5) \mu \mathrm{g} / \mathrm{L}$ for the heavy and occasional smokers, respectively. This difference between groups was highly significant $(p<0.0001)$. The highest $\mathrm{THCCOOH}$ concentration for the occasional smokers was below the LOQ value while the lowest concentration for the heavy smokers was $4.6 \mu \mathrm{g} / \mathrm{L}$. Similar results were obtained for $\mathrm{THCCOOH}$ levels just before smoking the placebo or the cannabis joint: the median $\mathrm{THCCOOH} \mathrm{C}_{0}$ were 12 and $20 \mu \mathrm{g} / \mathrm{L}$ for the heavy smokers and 0 and $0 \mu \mathrm{g} / \mathrm{L}$ for the occasional smokers, respectively. As described in the previous paragraph, $\mathrm{THCCOOH}$ concentrations measured $2.5 \mathrm{~h}$ after inhalation of the cannabis joint were 26 and $7.1 \mu \mathrm{g} / \mathrm{L}$ for the heavy and occasional smokers respectively. These values were also significantly different $(p<0.0001)$. Likewise, the difference between the $\mathrm{THCCOOH} \mathrm{Cmax}$ of the two groups was significant, with median values of 41 and $13 \mu \mathrm{g} / \mathrm{L}$ for heavy and occasional smokers, respectively. However, $\mathrm{THCCOOH} \mathrm{Cmax}$ determined in the blood of the occasional cannabis users were not significantly different from those measured in the heavy smokers' group before smoking the cannabis or the placebo joint. When we made the same comparison with the ranges of values measured at inclusion and at the end of the day of the experiment, significant differences were noticed between the heavy and occasional smokers $(p<0.0017$ and $<0.0023$ ).

\section{ROC curves before inhalation and after smoking}

In the ROC curves shown in Figure 3, the true positive rate (Sensitivity) is plotted as a function of the false positive rate (100-Specificity) for different cut-off points of the $\mathrm{THCCOOH}$ concentration. Each point on the ROC curve represents a sensitivity/specificity pair corresponding to a particular decision threshold. The main contribution of the ROC graph is to find out which $\mathrm{THCCOOH}$ concentration is the best decision threshold for differentiating occasional smokers from heavy users. Figure $3 a$ and $3 \mathrm{~b}$ present the $\mathrm{ROC}$ curves obtained with $\mathrm{THCCOOH}$ concentrations at $\mathrm{t}=0$, at $\mathrm{t}=2.5 \mathrm{~h}$ while Figure $3 \mathrm{c}$ displays the compilation of all time data, including those of the smoking session with placebo. The ROC curves of other sampling times are also calculated and show the same trends as those measured before smoking and at the end of the experiment (results not shown). The group of occasional smokers was selected as the control group while the heavy smokers were considered as the group being studied. The area under the ROC curve is a measure of how well a parameter can distinguish between the two groups of cannabis users (occasional/heavy). Areas of the ROC curves calculated for each sampling time are very similar, ranging from 0.90 to 0.96 , and thus can be considered as very informative. ${ }^{[19]}$ The same conclusion can be drawn from the compilation of all the time points (area equal to 0.88). Before inhalation, a sensitivity of $100 \%$ (i.e. including all the heavy users) was obtained for a THCCOOH concentration higher than $2.3 \mu \mathrm{g} / \mathrm{L}$. A $\mathrm{THCCOOH}$ level higher than $18.3 \mu \mathrm{g} / \mathrm{L}$ was associated with a 


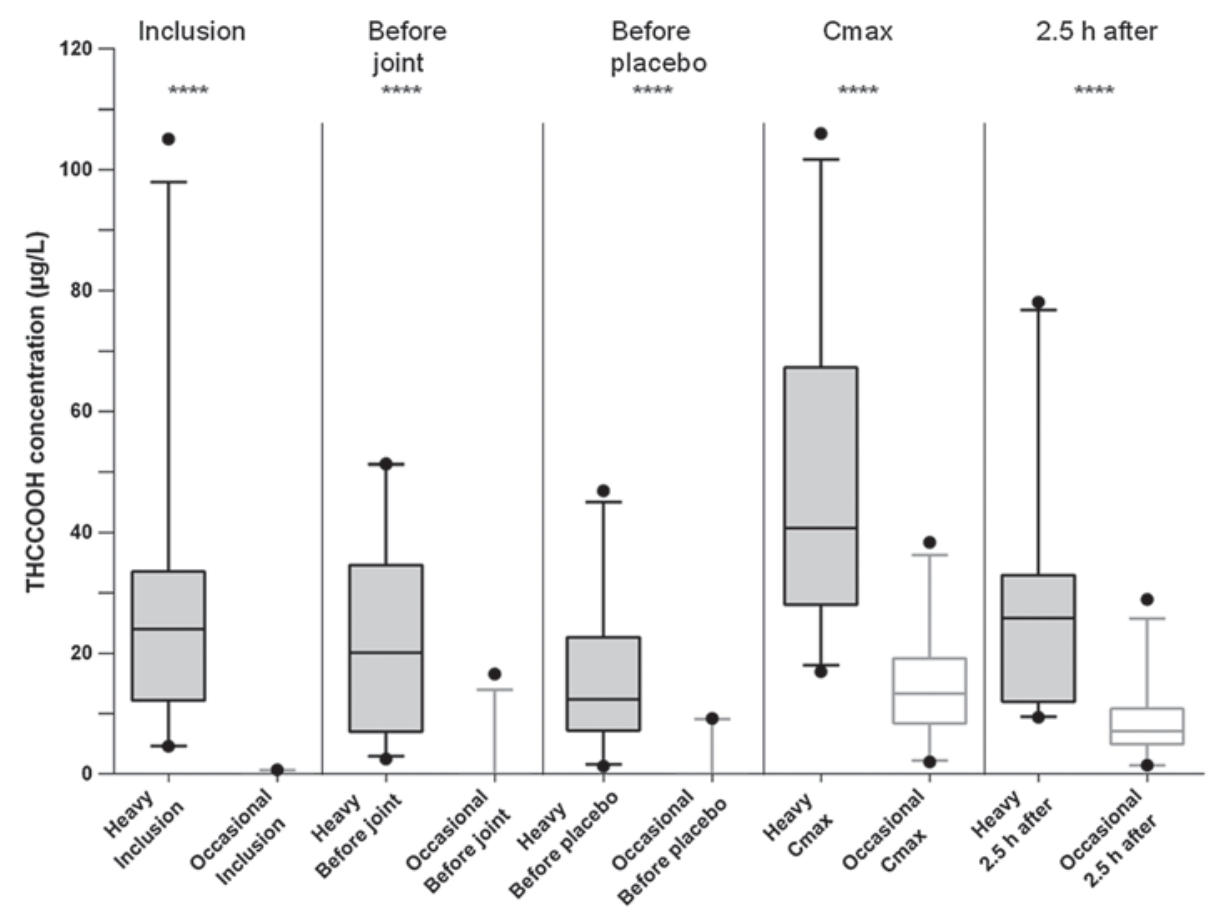

Figure 2. Box and whisker plots (5/95 percentile) comparing the distribution of THCCOOH concentrations in whole blood of occasional and heavy smokers at different times before and after smoking a cannabis or a placebo joint.

specificity of $100 \%$ (i.e. excluding all the occasional smokers). At $\mathrm{t}=2.5 \mathrm{~h}$, these cut-offs were $8.6 \mu \mathrm{g} / \mathrm{L}$ and $29.5 \mu \mathrm{g} / \mathrm{L}$ for a sensitivity and a specificity of $100 \%$, respectively. The two best decision thresholds (points 1 and 2, empty circles) were determined using the ROC curve that compiles all $\mathrm{THCCOOH}$ concentrations. Point 1 (lower left corner; $40 \mu \mathrm{g} / \mathrm{L}$ ) corresponds to the threshold with a specificity of $100 \%$ that is associated with the greatest sensitivity. This threshold ( $>40 \mu \mathrm{g} / \mathrm{L}$ ) is used to rule in the heavy smokers. Point 2 (towards the upper right corner; $3 \mu \mathrm{g} / \mathrm{L})$ is the threshold value that shows the closest sensitivity to $100 \%$ that is associated with the highest specificity. This threshold is used to rule out the heavy smokers.

To validate and confirm the choice of these two decision thresholds, their diagnostic characteristics were calculated and compared to those of other alternative cut-offs found on both sides of these values. Table 2 lists the sensitivity and specificity values with associated LR + obtained from the compilation of all time data. $\mathrm{THCCOOH}$ blood concentrations and corresponding serum values are also reported. This latter parameter was estimated from the $\mathrm{THCCOOH}$ serum/WB concentrations ratios determined by Giroud et al. (1.7) ${ }^{[20]}$ and corroborated by Karschner et al. (1.8). ${ }^{[21]}$ Table 2 indicates that the two selected cut-offs represent a good comprise between likelihood ratio, specificity, and sensitivity.

The $\mathrm{THCCOOH}$ threshold of $3.0 \mu \mathrm{g} / \mathrm{L}$ was correlated with a specificity of $62 \%(L R+$ of 2.6$)$ and a sensitivity of $97 \%$. The same parameters for a cut-off greater than $40 \mu \mathrm{g} / \mathrm{L}$ were 100 (infinite LR +) and $16 \%$. An infinite positive likelihood ratio indicated that the probability to be an occasional smoker with a $\mathrm{THCCOOH}$ level greater than $40 \mu \mathrm{g} / \mathrm{L}$ was null. Conversely, an LR + of 2.6 associated with a cut-off of $>3.0 \mu \mathrm{g} / \mathrm{L}$ indicated that it was only 2.6 times more likely to be a heavy smoker than an occasional user. Almost identical results were obtained when considering only the samples collected after cannabis smoking (results not shown). Table 3 lists the number of blood specimens that agreed or disagreed with the condition 'to be a heavy smoker' and fulfilled the requirement 'to be higher than the decision cut-off of 40 or $3 \mu \mathrm{g} \mathrm{THCCOOH} / \mathrm{L}^{\prime}$.

\section{Discussion}

$\mathrm{THCCOOH}$ concentrations in WB collected from documented heavy and occasional cannabis users enrolled in the controlled administration study are presented. The distinction between occasional and regular cannabis smokers is important in the context of driving assessment and evaluation of long-term fitness to drive. In this respect, Fergusson et al. ${ }^{[22]}$ have shown the correlation between the rate of active traffic accidents and an increased frequency of cannabis use. The main finding of our study was that the range of $\mathrm{THCCOOH}$ levels in the regular smokers was significantly higher than that in the occasional consumers. We also proposed two thresholds to help distinguish between regular and occasional cannabis users. According to Table 1, the two groups of smokers differed mostly in their frequency of cannabis consumption, with distinct and broad ranges of use. Because of the large variations in their smoking habits and inhalation techniques, a broad range of $\mathrm{THCCOOH}$ blood concentrations was expected at inclusion and throughout the day of the experiment. Although the concentrations varied considerably, $\mathrm{THCCOOH}$ blood levels between occasional and heavy smokers remained significantly different throughout the day of the experiment, as shown in Figure 1. It is noteworthy that the experimental conditions included a wide range of circumstances: blood sampled before smoking or after inhaling a placebo joint reflects what happens in the late elimination phase, while samples taken during the first $3 \mathrm{~h}$ following joint inhalation are typical of the distribution and early elimination phase. Furthermore, two methods of smoking are represented: $\mathrm{THCCOOH}$ detected at inclusion or after smoking a placebo is 

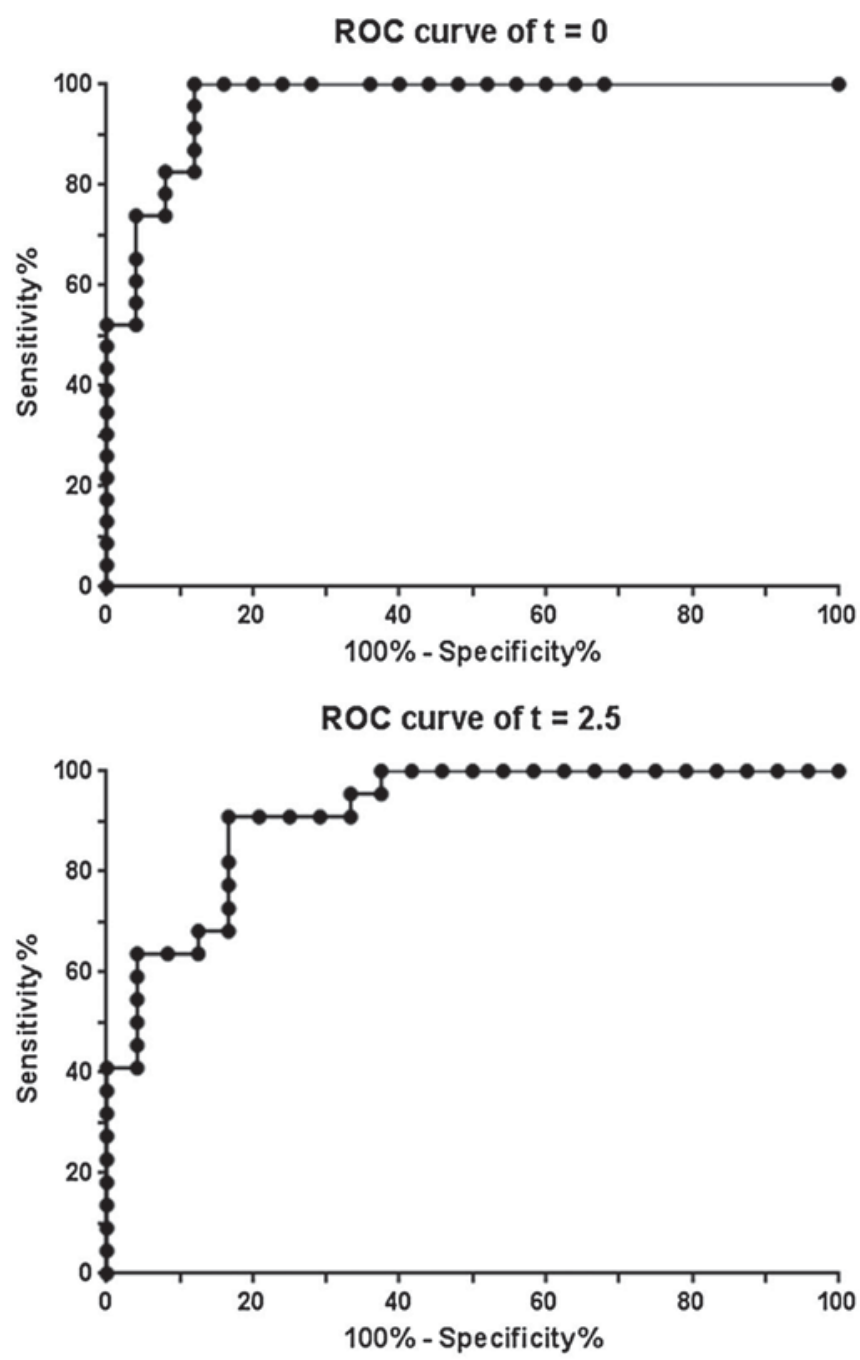

ROC curve : compilation of all the time values

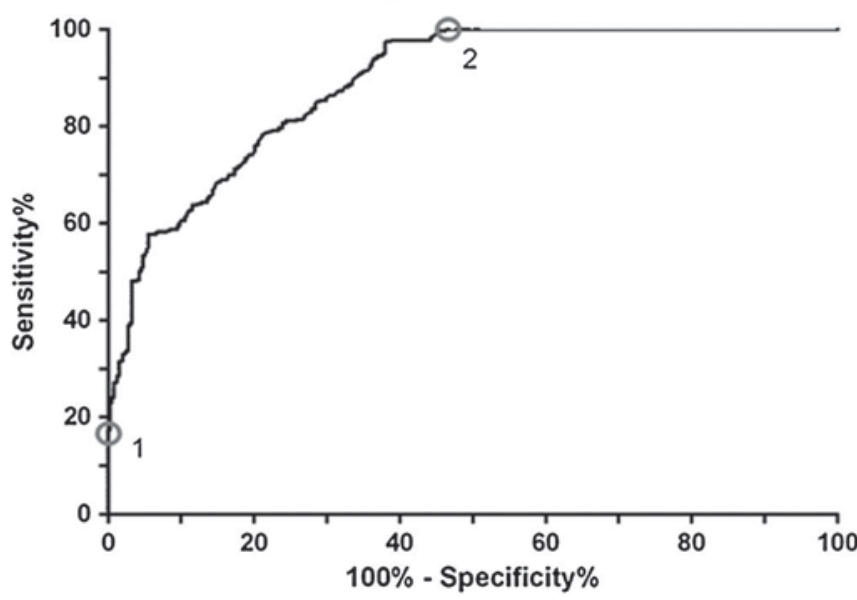

Figure 3. $\mathrm{ROC}$ curves of $\mathrm{THCCOOH}$ concentrations in whole blood calculated (a) before smoking $(\mathrm{t}=\mathrm{Oh}),(\mathrm{b})$ after smoking a cannabis joint $(\mathrm{t}=2.5 \mathrm{~h})$ and $(\mathrm{c})$ of THCCOOH values compiled throughout the study. Circle $1=40 \mu \mathrm{g} / \mathrm{L}$ : cut-off to rule in heavy smokers. Circle $2=3.0 \mu \mathrm{g} / \mathrm{L}$ : cut-off to rule out heavly smokers.

the result of ad libitum self-administration, while $\mathrm{THCCOOH}$ levels determined after joint smoking are the consequence of the controlled paced and self-administration. The differences observed in $\mathrm{THCCOOH}$ levels between the occasional and heavy smokers could therefore be used to establish indicators of the frequency of cannabis consumption. These differences were also found to be significant when considering each time point separately (see the box and whisker plots shown in Figure 2). However, as already mentioned by Toennes et al., ${ }^{[13]}$ the range of $\mathrm{THCCOOH}$ concentrations partially overlapped between each group. A grey area was found between about 3.0 and $30 \mu \mathrm{g} / \mathrm{L}$ for which no sharp distinction could be made. This can be observed in Figure 2; no significant difference could be made between $\mathrm{THCCOOH}$ Cmax in occasional smokers and $\mathrm{THCCOOH}$ concentrations measured in heavy smokers before smoking a joint or a placebo. Therefore, making a clear distinction between an occasional consumer who has just smoked a joint and a heavy smoker who has stopped smoking for several hours is hardly possible. Daldrup et al. ${ }^{[12]}$ suggested that $\mathrm{THCCOOH}$ and THC levels in serum could be used as indicators to discriminate between regular and occasional cannabis use. Their conclusions were that serum $\mathrm{THCCOOH}$ concentrations lower than $5 \mu \mathrm{g} / \mathrm{L}$ suggested occasional consumption and levels above $75 \mu \mathrm{g} / \mathrm{L}$ were correlated with regular consumption. These cut-offs were based on the few published studies that distinguish between regular and occasional consumers, and which also take into consideration the eight days between the relevant event and the time of blood sampling. Depending on whether the driver with a serum level of $75 \mu \mathrm{g} / \mathrm{L}$ stopped or continued to smoke, his serum concentration eight days before would range between 75 and $150 \mu \mathrm{g} / \mathrm{L}$. When taking into account the blood/ serum-plasma distribution of cannabinoids, ${ }^{[20,21]}$ corresponding WB concentrations of about 3, 43, and $86 \mu \mathrm{g} / \mathrm{L}$ can be calculated. As shown in Figure 2, this latter value greatly exceeds almost all of those determined for the group of heavy smokers. Similarly, the value of $43 \mu \mathrm{g} / \mathrm{L}$ exceeds most of the $\mathrm{THCCOOH}$ concentrations of the heavy smokers, while the proposed lowest threshold is in accordance with the majority of the values determined for the occasional smokers. Furthermore, none of the $\mathrm{THCCOOH}$ concentrations measured in the blood of the occasional smokers exceeded the threshold value of $43 \mu \mathrm{g} / \mathrm{L}$, while only a few values determined in the blood of the heavy smokers were lower than the $3 \mu \mathrm{g} / \mathrm{L}$ threshold. Our data were not inconsistent with the cut-off suggested by Daldrup et al. However, the experimental conditions of the two studies were significantly different. Regarding their usual consumption of cannabis, we did not ask the participants to change their habits. However, in the study of Daldrup et al., the subjects were suspected of driving under the influence of cannabis and were asked for further toxicological investigations within eight days to determine the serum $\mathrm{THCCOOH}$ concentrations. Although the terminal blood half-life of $\mathrm{THCCOOH}$ is very long, it can be assumed (especially if they were arrested in a time period preceding the terminal elimination phase) that they would have seriously decreased their consumption, resulting in higher levels of $\mathrm{THCCOOH}$ at the time of the event. Our study evaluates $\mathrm{THCCOOH}$ blood levels issues without exerting any influence on the smokers' behaviour. Participants were even encouraged to keep their habits unchanged and to maintain their usual cannabis consumption (frequency and dose), with the exception of abstinence during the four hours prior to the investigation day.

To determine our cut-off values, we established the ROC curves. The areas which were close to 1 indicated that heavy smokers could be clearly distinguished from occasional users. According to Table 2, if we select $3.0 \mu \mathrm{g} / \mathrm{L}$ as a cut-off (Point 2 
Table 2. Diagnostic characteristics of free $\mathrm{THCCOOH}$ concentrations test in cannabis smokers enrolled in a joint smoking administration study

\begin{tabular}{|lccccc|}
\hline & THCCOOH blood level & THCCOOH estimated serum level* & Sensitivity & Specificity & Likelihood ratio \\
\hline Heavy smokers rule out cut-off & $>2.5 \mu \mathrm{g} / \mathrm{L}$ & $>4.4 \mu \mathrm{g} / \mathrm{L}$ & 98 & 61 & 2.5 \\
& $>3.0 \mu \mathrm{g} / \mathrm{L}$ & $>5.2 \mu \mathrm{g} / \mathrm{L}$ & 97 & 62 & 2.6 \\
& $>5.0 \mu \mathrm{g} / \mathrm{L}$ & $>8.7 \mu \mathrm{g} / \mathrm{L}$ & 91 & 65 & 2.6 \\
Heavy smokers rule in cut-off & $>10 \mu \mathrm{g} / \mathrm{L}$ & $>17 \mu \mathrm{g} / \mathrm{L}$ & 75 & 80 & 3.7 \\
& $>30 \mu \mathrm{g} / \mathrm{L}$ & $>52 \mu \mathrm{g} / \mathrm{L}$ & 29 & 98 & 20 \\
& $>35 \mu \mathrm{g} / \mathrm{L}$ & $>61 \mu \mathrm{g} / \mathrm{L}$ & 23 & 99 & 90 \\
& $>40 \mu \mathrm{g} / \mathrm{L}$ & $>70 \mu \mathrm{g} / \mathrm{L}$ & 16 & 100 & $\infty$ \\
& $>45 \mu \mathrm{g} / \mathrm{L}$ & $>79 \mu \mathrm{g} / \mathrm{L}$ & 13 & 100 & $\infty$ \\
& $>50 \mu \mathrm{g} / \mathrm{L}$ & & & & \\
\hline
\end{tabular}

Table 3. Two by two tables for two different cut-offs ( 40 and $3.0 \mu \mathrm{g} / \mathrm{L})$. Number and percentage of blood specimens (total number $N=797$ ) which meet the condition "belong to the heavy smokers' group" and THCCOOH level "greater than the cut-off"

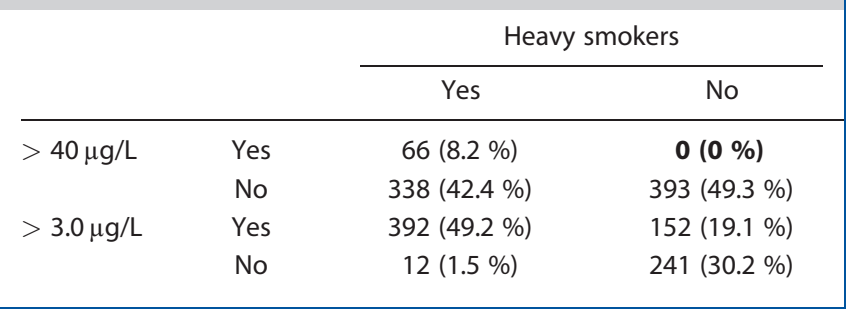

of the ROC curve), the specificity indicated that $62 \%$ of occasional smokers' $\mathrm{THCCOOH}$ values were correctly assigned to the occasional group. The sensitivity showed that $97 \%$ of the heavy users' samples were higher than $3.0 \mu \mathrm{g} / \mathrm{L}$ and were not falsely attributed to the occasional group. For an unknown sample with a $\mathrm{THCCOOH}$ level of $3.0 \mu \mathrm{g} / \mathrm{L}$, it was 2.6 times more likely that it belonged to the heavy smokers' group than to the occasional smokers. At a concentration of $40 \mu \mathrm{g} / \mathrm{L}$, only $16 \%$ of the heavy smokers' specimens were correctly detected, but no samples from the occasional consumers were falsely assigned (specificity of $100 \%$ ). This is confirmed by the results presented in Table 3. A null proportion of blood specimens from the occasional smokers presented a $\mathrm{THCCOOH}$ blood concentration higher than $40 \mu \mathrm{g} / \mathrm{L}$. Consequently, within the context of assessment of fitness to drive, our results suggest 3.0 and $40 \mu \mathrm{g} / \mathrm{L}$ as decision thresholds to assess in which category of cannabis smokers the drivers belong: occasional $(<3.0 \mu \mathrm{g} / \mathrm{L})$ or heavy smokers ( $>40 \mu \mathrm{g} / \mathrm{L})$ ? Our suggested cut-offs are quite similar to those proposed by Daldrup et al. ${ }^{[12]}$ An external validation of our decision thresholds was then carried out using the values reported by Toennes et al. ${ }^{[13]}$ In their study, the authors compared the serum cannabinoid pharmacokinetic properties in occasional and heavy users smoking a marijuana or placebo joint. Figure 4 shows the ROC curve obtained from these serum data that include 60 heavy smokers' samples and 22 specimens from occasional users. The area of the ROC curve $(0.7455)$ was much lower than that of our study. The sensitivity and specificity obtained with these data were $31 \%$ and $100 \%$, respectively, for a threshold of $70 \mu \mathrm{g} / \mathrm{L}$ of serum (corresponding to about $40 \mu \mathrm{g} / \mathrm{L}$ in WB, calculated with the serum-to-WB ratio of 1.75 ). This confirms that our proposed cut-off of $40 \mu \mathrm{g} / \mathrm{L}$ in WB can be used as a screening test to detect heavy cannabis users. Concerning the cut-off of $5 \mu \mathrm{g} / \mathrm{L}$ in serum (corresponding to $3 \mu \mathrm{g} / \mathrm{L}$ in WB),

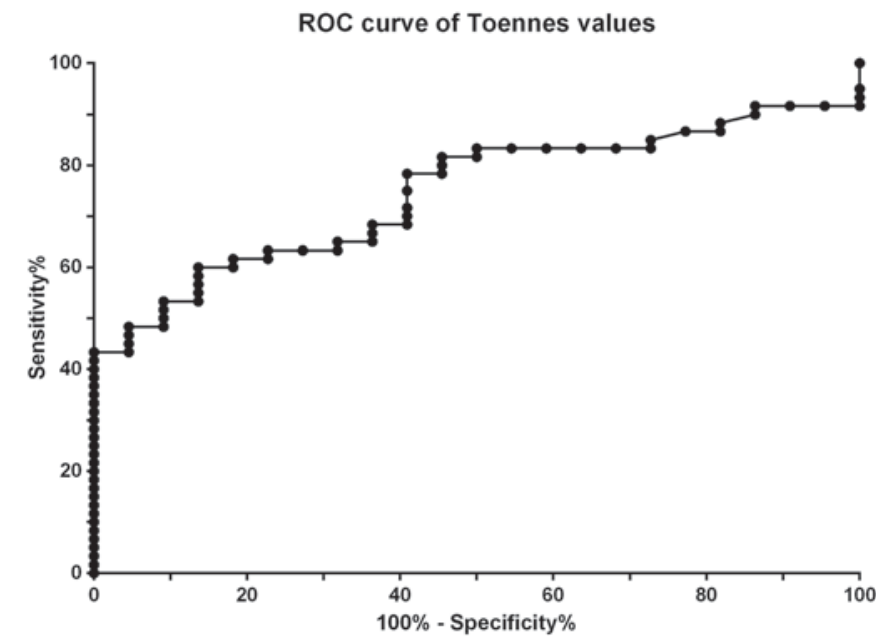

Figure 4. ROC curve established using values reported in Toennes et al.'s study ${ }^{[12]}$.

Toennes' values gave a specificity of $7.1 \%$ and a sensitivity of $92 \%$. However, because very few samples from the occasional smokers were below this threshold, the positive likelihood ratio was only 1.0. As mentioned before, there is a paucity of studies making a clear distinction between occasional and heavy smokers. Skopp and Pötsch ${ }^{[23]}$ showed that, 24-48 h after their last drug use, serum $\mathrm{THCCOOH}$ levels were below $17 \mu \mathrm{g} / \mathrm{L}$ in light users while heavy, regular users presented a free $\mathrm{THCCOOH}$ level reaching values of $297 \mu \mathrm{g} / \mathrm{L}$. If we consider the $\mathrm{THCCOOH}$ disposition in plasma of frequent cannabis users during continuous monitored abstinence, the results reported by Karschner et $a l .{ }^{[24]}$ indicate that after 5 days' abstinence none of 18 long-term heavy cannabis smokers were classified as heavy consumers according to our proposed decision threshold of $40 \mu \mathrm{g} / \mathrm{L}$ in WB. However, after so long a period of abstinence, these cannabis users can't be considered regular consumers anymore. In daily practice, a large-scale study carried out in all the main areas of Switzerland has shown that $45 \%$ of 6375 blood specimens collected during the years 2005 to 2007 were above the decision threshold of $40 \mu \mathrm{g} / \mathrm{L}$. The proportion of cases below $3 \mu \mathrm{g} / \mathrm{L}$ was insignificant (less than 1.5\%). ${ }^{[2,25]}$ Furthermore, the time interval between the traffic event and the blood sampling was unexpectedly short, with a mean value of $1.5 \mathrm{~h}$ (M. Augsburger, pers. comm.). This short period of time indicates that a retrospective calculation is generally not required. The circumstances in which the knowledge of the $\mathrm{THCCOOH}$ 
concentration can be of use are very specific. The detection of $\mathrm{THC}$ in the blood, within the context of the Swiss zero-tolerance policy, demonstrates that at the time of blood sampling the driver was under the influence of cannabis and his/her ability to drive was impaired short-term. The forensic expert is then asked to determine whether their fitness to drive is also altered long-term. Depending on the clinical and psychological context of the driver, knowledge of blood $\mathrm{THCCOOH}$ levels may be very useful to guide or narrow diagnosis of chronic use and possibly of dependence, and facilitate therapeutic follow-up and treatment. A long and costly therapeutic monitoring will be not necessary if the $\mathrm{THCCOOH}$ level is below $3 \mu \mathrm{g} / \mathrm{L}$ and the medical interview suggests that cannabis smoking is occasional at most. In this case, the driver can reclaim their driving license. On the other hand, if the $\mathrm{THCCOOH}$ level is higher than $40 \mu \mathrm{g} / \mathrm{L}$ and the medical assessment indicates a regular consumption, a therapeutic follow-up for a cannabis use disorder must be undertaken. After a proven period of abstinence and regular medical follow-ups, a driving license may be returned to a driver who has been a heavy cannabis smoker in the past. If the $\mathrm{THCCOOH}$ concentration is in the grey zone between 3 and $40 \mu \mathrm{g} / \mathrm{L}$ and the medical assessment does not exclude a regular use, it is advisable to undertake a forensic expertise and a therapeutic follow-up. Therefore, THCCOOH provides a useful orientation tool for the forensic expert within the context of assessing fitness to drive.

\section{Limitations of the study}

For each clinical trial, compromises and choices must be made. In this broad cannabis administration study, two parameters have greatly influenced the time-schedule of the day of experiment. First, we chose to keep the volunteers under close supervision for several hours before administration of the joint. The second parameter was the timing of the fMRI sessions. Finally, the infrastructure of the clinical research centre, the ethical committee agreement, and the volunteers' availability were not compatible with a multi-day experiment. The drawback was that a long investigation after inhalation was not possible. The robustness of the proposed cut-offs should be tested in a variety of conditions. Other ways of smoking and different dosage regimens should be investigated. The influence of varying the proportions of tobacco should also be tested. Furthermore, different frequencies of consumption could be considered, for instance regular versus intermittent versus 'weekend binge weed smoking'. In addition, the concurrent intake of illegal or therapeutic drugs interacting with THC metabolism could be studied. The consequences of altered THC metabolism related to genetic polymorphisms of CYP2C9 and glucuronosyltransferase enzymes should also be evaluated. In our opinion, it could be interesting to quantify separately free $\mathrm{THCCOOH}$ and its glucuronide conjugate. However, this molecule is almost never considered in routine work.

\section{Conclusion}

Whole blood THCCOOH concentrations of heavy cannabis smokers determined in a controlled administration study greatly exceeded those measured in the occasional users' group. Therefore, we can take advantage of this difference to estimate to which category of smokers the drivers belong. A level of $\mathrm{THCCOOH}$ higher than
$40 \mu \mathrm{g} / \mathrm{L}$ is strongly correlated with a heavy consumption of cannabis, whereas a concentration below $3 \mu \mathrm{g} / \mathrm{L}$ suggests occasional use. These threshold values can be used as guidance criteria to help the forensic expert in the classification of impaired drivers as heavy or occasional users. A heavy consumption may suggest a long-term unfitness to drive that should be followed by further toxicological investigations and therapeutic follow-up. Once abstinence has been demonstrated, the driver can reclaim their driving license.

\section{Acknowledgements}

The authors would like to thank the Swiss National Science Foundation (FNS_320030_127507/1), the Centre d'Imagerie BioMédicale (CIBM) and the Faculty of Biology and Medicine (interdisciplinary grant) at the University of Lausanne for their financial support. We thank Dr Marc Augsburger of the University Center of Forensic Medicine Lausanne-Geneva, Prof. Thierry Buclin, and the staff of the division of Clinical Pharmacology and Toxicology at CHUV. We also thank Dr Frank Sporkert for fruitful discussion.

\section{References}

[1] http://www.bag.admin.ch/suchtmonitoring/index.html?lang=fr. Monitoring suisse des addictions: être bien informé pour mieux prévenir. [October 2013]

[2] M.C. Senna, M. Augsburger, B. Aebi, T.A. Briellmann, N. Donze, J.L. Dubugnon, P.X. Iten, C. Staub, W. Sturm, K. Sutter. First nationwide study on driving under the influence of drugs in Switzerland. Forensic Sci. Int. 2010, 198, 11.

[3] M. Augsburger, N. Donze, A. Menetrey, C. Brossard, F. Sporkert, C. Giroud, P. Mangin. Concentration of drugs in blood of suspected impaired drivers. Forensic Sci. Int. 2005, 153, 11.

[4] A. Menetrey, M. Augsburger, C. Giroud, P. Mangin. [Cannabis and automobile driving]. Praxis (Bern 1994) 2001, 90, 1398.

[5] M. Fabritius, H. Chtioui, G. Battistella, J.M. Annoni, K. Dao, B. Favrat, E. Fornari, E. Lauer, P. Maeder, C. Giroud. Comparison of cannabinoid concentrations in oral fluid and whole blood between occasional and regular cannabis smokers prior and after smoking a cannabis joint. 2013, Anal. Bioanal. Chem, DOI: 10.1007/s00216-013-7412-1.

[6] J.G. Ramaekers, G. Kauert, E.L. Theunissen, S.W. Toennes, M.R. Moeller. Neurocognitive performance during acute THC intoxication in heavy and occasional cannabis users. J. Psychopharmacol. 2009, 23, 266.

[7] B. Moosmann, N. Roth, V. Auwarter. Hair analysis for THCA-A, THC and $\mathrm{CBN}$ after passive in vivo exposure to marijuana smoke. Drug Test. Anal. 2013, DOI: 10.1002/dta.1474.

[8] M.A. Huestis, R.A. Gustafson, E.T. Moolchan, A. Barnes, J.A. Bourland, S.A. Sweeney, E.F. Hayes, P.M. Carpenter, M.L. Smith. Cannabinoid concentrations in hair from documented cannabis users. Forensic Sci. Int. 2007, 169, 129.

[9] F. Musshoff, B. Madea. Review of biologic matrices (urine, blood, hair) as indicators of recent or ongoing cannabis use. Ther. Drug Monit. 2006, 28, 155.

[10] E. Han, H. Choi, S. Lee, H. Chung, J.M. Song. A study on the concentrations of 11-nor-Delta(9)-tetrahydrocannabinol-9-carboxylic acid (THCCOOH) in hair root and whole hair. Forensic Sci. Int. 2011, 210, 201.

[11] E. Han, H. Chung, J.M. Song. Segmental hair analysis for 11-nor-Delta (9)-tetrahydrocannabinol-9-carboxylic acid and the patterns of cannabis use. J. Anal. Toxicol. 2012, 36, 195.

[12] T. Daldrup, H. Käferstein, H. Köhler, R. Maier, F. Musshoff. Deciding between one off/occasional and regular cannabis consumption. Blutalkohol 2000, 37, 39.

[13] S.W. Toennes, J.G. Ramaekers, E.L. Theunissen, M.R. Moeller, G.F. Kauert. Comparison of cannabinoid pharmacokinetic properties in occasional and heavy users smoking a marijuana or placebo joint. J. Anal. Toxicol. 2008, 32, 470.

[14] Société Française de Toxicologie Analytique. Website. Available at: http:// sfta.org/presentation/main/main_accueil.php [24 October 2013]

[15] G. Battistella, E. Fornari, A. Thomas, J.F. Mall, H. Chtioui, M. Appenzeller, J.M. Annoni, B. Favrat, P. Maeder, C. Giroud. Weed or wheel! FMRI, behavioural, and toxicological investigations of how cannabis smoking affects skills necessary for driving. PLoS One 2013, 8, e52545. 
[16] A. Thomas, C. Widmer, G. Hopfgartner, C. Staub. Fast gas chromatography and negative-ion chemical ionization tandem mass spectrometry for forensic analysis of cannabinoids in whole blood. J. Pharm. Biomed. Anal. 2007, 45, 495.

[17] M. Fabritius, C. Staub, P. Mangin, C. Giroud. Analysis of cannabinoids in oral fluid by liquid chromatography-tandem mass spectrometry. Forensic Toxicol. 2013, 31, 151.

[18] L. Chrostek, B. Cylwik, E. Gruszewska, J. Tobolczyk. The diagnostic power of direct carbohydrate-deficient transferrin immunoassay in alcoholics. Absolute or relative values? Alcohol 2012, 46, 69.

[19] H. Delacour, A. Servonnet, A. Perrot, J.F. Vigezzi, J.M. Ramirez. [ROC (receiver operating characteristics) curve: principles and application in biology]. Ann. Biol. Clin. (Paris) 2005, 63, 145.

[20] C. Giroud, A. Menetrey, M. Augsburger, T. Buclin, P. Sanchez-Mazas, P. Mangin. Delta(9)-THC, 11-OH-Delta(9)-THC and Delta(9)-THCCOOH plasma or serum to whole blood concentrations distribution ratios in blood samples taken from living and dead people. Forensic Sci. Int. 2001, 123, 159.
[21] E.L. Karschner, D.M. Schwope, E.W. Schwilke, R.S. Goodwin, D.L. Kelly, D.A. Gorelick, M.A. Huestis. Predictive model accuracy in estimating last Delta9-tetrahydrocannabinol (THC) intake from plasma and whole blood cannabinoid concentrations in chronic, daily cannabis smokers administered subchronic oral THC. Drug Alcohol Depend. 2012, 125, 313.

[22] D.M. Fergusson, L.J. Horwood. Cannabis use and traffic accidents in a birth cohort of young adults. Accid. Anal. Prev. 2001, 33, 703.

[23] G. Skopp, L. Potsch. Cannabinoid concentrations in spot serum samples 24-48hours after discontinuation of cannabis smoking. J. Anal. Toxicol. 2008, 32, 160.

[24] E.L. Karschner, E.W. Schwilke, R.H. Lowe, W.D. Darwin, R.I. Herning, J.L. Cadet, M.A. Huestis. Implications of plasma Delta9tetrahydrocannabinol, 11-hydroxy-THC, and 11-nor-9-carboxy-THC concentrations in chronic cannabis smokers. J. Anal. Toxicol. 2009, 33, 469.

[25] SGRM. Description de la section. Available at: http://www.sgrm.ch/fr/ chemie/ueber-die-sektion.html [24 October 2013]. 